\title{
Research on Multi-objective Algorithm of Air Defense Missile Fire Unit
}

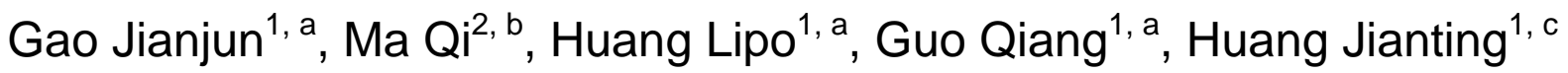 \\ ${ }^{1}$ Infrastructure Department, Air Defense Forces Academy, Zhengzhou, 450052, China \\ ${ }^{2}$ Department of scientific research, Air Defense Forces Academy,Zhengzhou, 450052, China \\ aemail: jjgao1022@163.com, bemail:jsycjys@163.com, cemail:ttbear113@163.com
}

Keywords: Target Interception; Favorable Degrees; Anti-aircraft missiles; Fire units; Algorithm

\begin{abstract}
Intercept sorting is by targets recognition and the threat judgment, targets is determined as the intercept object, which not only reflects the target threat degree, but also reflects the degree of emergency for interception target, target interception order can provide a basis for objective optimization, so as to make the necessary preparation for target interception. Through the analysis of the intercept related factors of advantage degree, combining the actual circumstance of attacking target, two targets to be studied for all targets to be killed with the shortest time and the shortest intercept total time. We established the decision-making model for multi-channel anti-aircraft missile fire units, given interception decision algorithm, and considered the complexity of the algorithm, derived model and algorithm is more scientific and reasonable, which will provide ideas for effective interception.
\end{abstract}

\section{Introduction}

In the traditional command conditions, fire distribution mainly relys on the commanders for decision-making, while the commander can only be analyzed based on past experience; and under the conditions of command automation, the fire distribution assisted decision-making by computer, threat degrees and intercept favorable degree can be accuratly calculated and sorting in a very short time, and in accordance with the appropriate mathematical model to optimize the allocation of firepower and choose the program which can maximize the effectiveness ${ }^{[1]}$. In fire distribution, the most important thing is to reasonablly intercept air targets, this problem is currently to follow some criteria, but faced with the complexity of the air situation, the target characteristic diversity, corresponding to different interception strategy, Therefore, it should be based on an objective function, establish mathematical model and design or take the appropriate algorithms, in order to reach the optimal intercept effect, thereby increasing the effectiveness of firepower distribution.

Target threat evaluation and interception sorting are the key sub-processes in shooting command and control process of air defense missile. With the development of air defense systems, domestic and foreign scholars have been many studies on interception sorting criteria, some criteria even antagonistic, the literature [2] analyzed these cases in detail, the literature [3] pointed out that a reasonable interception order should be based on the actual target situation and the number of air defense weapon system. In this paper, a mathematical model is established ,which discussed multi-channel air defense missile system to intercept targets scheduling problem, research under the premise of killing all targets with the shortest time, make the shortest total time for each channel.

\section{Interception opportune factor and condition}

In the anti-air war, multiple fire units and multiple batches targets should be calculated separately for the favorable extent, in order to obtain a reasonable interception scheme for maximum interception performance. Factors affecting interception favorable degree are the following: the maximum effective interception time, altitude, shortcut route projection relative to each fire unit, flight speed, the order of firepower units were crossing, aerial targets types, and air defense weapon system tactical and technical indicators and other factors ${ }^{[4]}$. and acording to the target route distance can calculate the maximum effective interception time, the target route 
distance refers to the target in route current position to defend the target horizontal distance, is the main basis for the determination of air defense missile troops fighting level and weapon system ready class. The target route distance closer descript army combat need be ready to higher levels and the greater target threat degree. Cast electronic interference also affect the guidance flight of air defense missile, reduce the other combat effectiveness, an important means to improve their viability. So, the enemy air raid weapon electronic interference ability is an important factor of target interception opportune degree.

A target channel intercepts two continuous follow-up of air target, which need meet the following conditions $^{[4]}$ :

$$
\Delta D_{L S 2} \geq\left(\frac{\Delta D_{f y 1}}{v_{m 1}}+d_{m z y 1}+2 t_{e}+t_{p}+t_{z}\right) v_{m 2}
$$

and $\Delta D_{L S 2}$ as the second air target corresponding to the near boundary distance, namely ,air target 2 intercept remaining distance; $\Delta D_{f y 1}$ is the first air targets corresponding to far boundary distance of emission region; $d_{m 1}$ is the flying speed of the first air targets; $d_{m z y 1}$ is the encounter time of the air defense missile and aerial target 1 in the far field of kill zone; $t_{e}$ is a burst missile launching interval sum; $t_{p}$ is an aerial target shooting results evaluation time; $t_{z}$ is the firing transfer time; $v_{m 2}$ is the second air targets flying speed. Can also be written as a time expression:

$$
t_{l s g 2} \geq t_{l s y 1}+t_{m y y 1}+2 t_{e}+t_{p}+t_{z}
$$

And $t_{l s g 2}$ as intercept remaining time of air target 2; $t_{l s y 1}$ is the time of reaching far boundary of emission region for the first air targets.

\section{Modeling assumptions}

(1) the number of air defense missile weapon systems are limited, but there is enough bombs.

(2) After the first interception, command and control systems can continue to turn the fire, implement of interception target not to be killed.

(3) In the interception, no two or more than two channel intercept the same target.

(4) As our air defense weapon systems are adequate, all targets can intercept can be damage.

\section{Intercept sorting model}

Set targets set $M=\left\{M_{1}, M_{2}, \cdots, M_{n}\right\}$, our air defense missile weapon system consists of $m$ channels, $M_{j}(j=1,2, \cdots, n)$ enters the kill zone at a time $t_{j}^{1}$, and might bomb after the time $t_{j}^{2}$, so we must kill the target $M_{j}$ in the time interval $\left[t_{j}^{1}, t_{j}^{2}\right]$, and set the interception the remaining time of goal $M_{j}(j=1,2, \cdots, n)$ is $t_{l s g j}=t_{j}^{2}-p_{i j}(j=1,2, \cdots, n)$ at the moment of decision. when channel $i$ intercept the target $M_{j}$, fired missiles at time $s_{i j}$, missile flight time is $p_{i j}$, the encounter timeis $c_{i j}$, meeting $c_{i j}=s_{i j}+p_{i j}$. Because the target $M_{j}(j=1,2, \cdots, n)$ must be killed within time range $\left[t_{j}^{1}, t_{j}^{2}\right]$, so need began firing after $t_{j}^{1}-p_{i j}$, finish firing before $t_{l s g j}-p_{i j}$, that is $t_{j}^{1}-p_{i j} \leq s_{i j} \leq t_{l s g j}-p_{i j}$. Determining an interception scheme (interception order), which make the shortest total time for each channel under the premise of killing all targets with the shortest time.

\section{Related definitions and theorem}

Define1 all the nodes in the network are arranged in a matrix form, the $j$ column nodes only get to the $j+1$ column nodes, the nodes are empowered as the interception time, and the edge is not empowered, which is called as the intercept Matrix network. 
Definition 2 according to intercept matrix network infrastructure, the path begins from the nodes of the first column to the nodes of the end column, called as a node path of the intercept matrix network. The values of all nodes on the node road are called as a node path length value. the weights of the peer nodes on node path constitute vector referred to interception node path vector.

Define3 set $d=\left(d_{1}, d_{2}, \cdots, d_{m}\right)^{T}$, then call $\max \left\{d_{1}, d_{2}, \cdots, d_{m}\right\}$ as the wide of vector $d$, denoted by $[d]^{[5]}$.

Define4 set $d=\left(d_{1}, d_{2}, \cdots, d_{m}\right)^{T}$, then call $\sum_{i=1}^{m} d_{i}$ as the quality of vector $d$, denoted by $\langle d\rangle$.

Define5 Set $\left(\begin{array}{l}d_{1} \\ d_{2} \\ \vdots \\ d_{i} \\ \vdots \\ d_{m}\end{array}\right) \oplus p_{i j}=\left(\begin{array}{l}d_{1} \\ d_{2} \\ \vdots \\ d_{i}+p_{i j} \\ \vdots \\ d_{m}\end{array}\right) \quad, \quad$ then called as vector plus of $d=\left(d_{1}, d_{2}, \cdots, d_{i}, \cdots, d_{m}\right)^{T}$ and the number $p_{i j}$.

Theorem time of killing all target $=\min \{$ the wide of node path vector $\}$, Total time $=$ interception node path length value.

the intercept matrix network, if nodes are weighted value for $p_{i j}(i=1,2, \cdots, m ; j=1,2, \cdots, n)$, by the introduction of the above concept, the target intercept problem can be regarded as a kind of network optimal path problem: in all nodes paths of the least nodes paths wide, find a minimum node path length value and the most favorable interception node road, the corresponding program is the scheme with the shortest total time for each channel under the premise of killing all targets with the shortest time.

\section{Algorithm}

step1 Calculat intercept remaining time of the target can be intercepted $t_{\text {lsgj }}(j=1,2, \cdots, n)$, according to the conditions of intercepting two continuous target,

If $t_{\text {lsgi }} \geq t_{\text {lsyi }_{k-1}}+t_{m z y i_{k-1}}+2 t_{e}+t_{p}+t_{z}$, turn to step 2;

If $t_{\text {lsgi }_{k}}<t_{\text {lsyi }_{k-1}}+t_{m z y i_{k-1}}+2 t_{e}+t_{p}+t_{z}$, reject $J_{i_{k}}$, give up interception.

step 2 Note interception matrix network composed by the target can be intercepted

$$
\left(\begin{array}{cccc}
p_{11} & p_{12} & \cdots & p_{1 n} \\
p_{21} & p_{22} & \cdots & p_{2 n} \\
\cdots & \cdots & \cdots & \cdots \\
p_{m 1} & p_{m 2} & \cdots & p_{m n}
\end{array}\right)
$$

initial labeling $q_{i 1}=\left(0, \cdots, p_{i 1}, \cdots, 0\right)^{T}, \quad q_{i j} \Leftarrow(\infty, \infty, \cdots, \infty)^{T}(i=1,2, \cdots, m ; j=1,2, \cdots, n)$, the initial pointer matrix $l(i, j) \Leftarrow(i, j)(i=1,2, \cdots, m ; j=1,2, \cdots, n)$.

step 3 set $i \Leftarrow 1, \quad j \Leftarrow 1, \quad k \Leftarrow 1$.

step 4 Calculat vector addition and comparison with vector width,if $\left[q_{i k} \oplus p_{j, k+1}\right]<\left[q_{j, k+1}\right]$, then $q_{j, k+1} \Leftarrow q_{i k} \oplus p_{j, k+1}, l(j, k+1) \Leftarrow l(i, k)$, turn to step 6; otherwise $\left[q_{i k} \oplus p_{j, k+1}\right] \geq\left[q_{j, k+1}\right]$, turn to step 5 . step 5 If $\left[q_{i k} \oplus p_{j, k+1}\right]=\left[q_{j, k+1}\right]$, and $\left\langle q_{i k} \oplus p_{j, k+1}\right\rangle \leq\left\langle q_{j, k+1}\right\rangle$, 
then $q_{j, k+1} \Leftarrow q_{i k} \oplus p_{j, k+1}, \quad l(j, k+1) \Leftarrow l(i, k) \quad$ (as $\left\langle q_{i k} \oplus p_{j, k+1}\right\rangle=\left\langle q_{j, k+1}\right\rangle$, priority to intercept the most favorable targets ) turn to step 6; otherwise $\left[q_{i k} \oplus p_{j, k+1}\right] \geq\left[q_{j, k+1}\right], \quad q_{j, k+1}$ and $l(j, k+1)$ are the same, turn to step 6 .

step 6 If $j<m$, then $j \Leftarrow j+1$, turn to step 4; otherwise $(j=m)$, turn to step 7 .

step 7 If $i<m$, then $i \Leftarrow i+1, j \Leftarrow 1$, turn to step 4; otherwise ( $i=m$ ), turn to step 8 .

step 8 If $k<n-1$, then $k \Leftarrow k+1, i \Leftarrow 1, j \Leftarrow 1$, turn to step 4; otherwise $(k=n-1)$, turn to step 9.

step 9 Calculat $\min _{1 \leq i \leq m}\left\{\left[q_{i, n}\right]\right\} \stackrel{\Delta}{=}\left[q_{\lambda n}\right]$, Output optimal target interception scheme $Z$ :

$$
(\lambda, n), l(\lambda, n) \stackrel{\Delta}{=}\left(\lambda_{1}, n-1\right), \cdots, l\left(\lambda_{n-2}, 2\right) \stackrel{\Delta}{=}\left(\lambda_{n-1}, 1\right) .
$$

step10 According to the optimal target interception program $Z$ to intercept, when a new target appears or not to damage the original target, and there is an idle channel turn to step step 1.

\section{Conclusion}

In raids and counter-raids combat, air defense system can not fully grasp the composition of the pre-raid system, incoming targets with diverse characteristics and fickle, the enemy air attack weapons attacking time and approach, etc. are dynamic, uncertain, and therefore air targets in real time, reasonable intercept order is extremely important. In this paper, by establishing a mathematical model, constructing matrix network, make sure our air defense missile fire units to intercept the target program, making the shortest total time for each channel under the premise of killing all targets with the shortest time. However, this article does not take the threaten degree as the objective function, in the air attack and air defense combat process, due to intercept the air targets of a larger threat value, equivalent to intercept a plurality of general air targets, so should be the first to intercept the largest threat air targets, in order to make my defense weapon system intercepting biggest effectiveness. In the future it is necessary to take the threaten degree as a target in the multi-objective to be studied in order to improve the applicability of the model.

\section{Reference}

[1] Lou Shouchun. Air defense missile firing command control model [M]. Beijing: national defence industry press, 2009.

[2] Xu Gao-ping, “Air defense missile fire units for air target interception scheduling problem”, Tactical Missile Technology China, vol. 6, Feb. 2007, pp. 01-09.

[3] GaoJianjun,Guo Qiang. Air defense missile fire units intercept Ordination under the conditions of specified target without delay [J]. Journal of Air Defense Forces Academy, 2013(4): 53-54.

[4] Jiang Liqiang. Air defense combat simulation method [M]. Beijing: Ocean Press, 2009.

[5] Fu Xiaowei, Guo Qiang, Ma Qinqin. For a class of uncertain Multi-objective Assignment Problem and algorithm [J]. operations research and management Science,2013, pp. 34-38. 